\title{
Cognitive flexibility in autism spectrum disorder: Explaining the inconsistencies?
}

\author{
Lien Van Eylen ${ }^{\mathrm{a}, \mathrm{b}, \mathrm{c}, \mathrm{d}, *}$, Bart Boets ${ }^{\mathrm{b}, \mathrm{d}}$, Jean Steyaert ${ }^{\mathrm{b}, \mathrm{d}, \mathrm{f}}$, Kris Evers ${ }^{\mathrm{b}, \mathrm{c}, \mathrm{d}}$, \\ Johan Wagemans ${ }^{\mathrm{c}, \mathrm{d}}$, Ilse Noens ${ }^{\mathrm{a}, \mathrm{d}, \mathrm{e}}$ \\ a Parenting and Special Education Research Group, Katholieke Universiteit Leuven (K.U. Leuven), Belgium \\ ${ }^{\mathrm{b}}$ Department of Child Psychiatry, UPC-K.U. Leuven, Belgium \\ ${ }^{\mathrm{c}}$ Laboratory of Experimental Psychology, K.U. Leuven, Belgium \\ ${ }^{\mathrm{d}}$ Leuven Autism Research (LAuRes), K.U. Leuven, Belgium \\ e Psychiatric and Neurodevelopmental Genetics Unit, Massachusetts General Hospital, Boston, USA \\ ${ }^{\mathrm{f}}$ Department of Clinical Genetics, University Hospital Maastricht, The Netherlands
}

\section{A R T I C L E I N F O}

\section{Article history:}

Received 23 January 2011

Accepted 25 January 2011

Available online 20 February 2011

\section{Keywords:}

Autism spectrum disorders

Cognitive flexibility

Task-switching

Wisconsin Card Sorting Task

Disengagement

Task instructions

\begin{abstract}
A B S T R A C T
The Wisconsin Card Sorting Task (WCST) is the only cognitive flexibility task that has consistently shown deficits in individuals with an autism spectrum disorder (ASD). As this is the only task characterized by limited explicit task instructions and a high degree of disengagement required to perform the switch, we hypothesized that cognitive flexibility deficits of individuals with ASD might only become apparent in situations fulfilling these requirements. However, the WCST involves various additional cognitive processes besides switching, making it uncertain whether difficulties are indeed due to cognitive flexibility impairments. The aim of this study was to investigate whether individuals with ASD show cognitive flexibility impairments on a more controlled task-switching variant of the WCST, still fulfilling both requirements. We therefore developed such a task and administered it to 40 high-functioning children with ASD and 40 age- and IQ- matched typically developing controls. As predicted, individuals with ASD made more perseveration errors and had a significantly higher switch cost than typically developing controls, but they performed equally well on the control measures.
\end{abstract}

(c) 2011 Elsevier Ltd. All rights reserved.

\section{Introduction}

Autism spectrum disorders (ASDs) are early onset neurodevelopmental disorders characterized by a co-occurrence of impairments in social reciprocity and communication, combined with restricted and repetitive patterns of interests and activities (American Psychiatric Association [APA], 2000). These restricted and repetitive behaviors and interests have been proposed to be associated with executive dysfunctions in individuals with ASD (Boyd, McBee, Holtzclaw, Baranek, \& Bodfish, 2009; Happé \& Ronald, 2008; Hill, 2004; Turner, 1997). Executive functioning (EF) involves goal-oriented planning and regulation of thoughts and actions (Denckla, 1996). It is an umbrella term for several higher-order cognitive functions (Hill, 2004). Pennington and Ozonoff (1996) have outlined six EF domains: inhibition, working memory, contextual memory, planning, fluency (or generativity), and cognitive flexibility (or set-shifting). Of all these EF domains, cognitive flexibility has been most clearly related to repetitive behaviors in ASD (Lopez, Lincoln, Ozonoff, \& Lai, 2005).

\footnotetext{
* Corresponding author at: Department of Child Psychiatry, University Hospital of Leuven, Herestraat 49 - Box 7003, B-3000 Leuven, Belgium. Tel.: +32(0)16/34 2245 .

E-mail address: Lien.VanEylen@ped.kuleuven.be (L. Van Eylen).
} 
Cognitive flexibility refers to "the ability to shift to different thoughts or actions depending on situational demands" (Geurts, Corbett, \& Solomon, 2009, p. 74). Although it has been shown that flexibility deficits in ASD do occur and that they are related to repetitive behaviors in ASD (South, Ozonoff, \& McMahon, 2007; Yerys et al., 2009), there are many inconsistent findings. Studies investigating cognitive flexibility in natural settings by means of the Behavior Rating Inventory of Executive Function (BRIEF) have shown that people with ASD have problems with flexibility in daily life (Gioia, Isquith, Kenworthy, \& Barton, 2002; Mackinlay, Charman, \& Karmiloff-Smith, 2006). These flexibility deficits have also been related to the repetitive behaviors typical for ASD (Boyd et al., 2009). However, studies measuring cognitive flexibility in a clinical or research setting have yielded more inconsistent findings.

Table 1 provides an overview of the most commonly used cognitive flexibility tasks in autism research, indicating the number of studies showing deficient versus intact performance in ASD. In a recent review, Geurts et al. (2009) make a distinction between three types of cognitive flexibility tasks: (a) traditional clinical neuropsychological measures, (b) a hybrid neuropsychological/experimental paradigm (i.e., the intra-dimensional/extra-dimensional shift task [ID/ED] of the Cambridge Automated Neuropsychological Test and Battery - CANTAB), and (c) experimental task-switching paradigms. Firstly, the clinical neuropsychological measures are mostly paper-and-pencil tasks, without control stages measuring possible confounding variables. Concerning these measures, all studies using the Wisconsin Card Sorting Task (WCST) have consistently reported deficits in individuals with ASD, whereas the majority of studies using other neuropsychological measures (e.g., the trail making test) reported intact performance. Secondly, the ID/ED shift task is a computerized task that contains several stages measuring possible confounds. Studies using this task also yielded inconsistent findings, with the majority of studies showing intact performance in individuals with ASD (as measured by the number of errors on the EDshift trials). Thirdly, the experimental task-switching paradigms provide a more controlled measure of cognitive flexibility by allowing the calculation of a switch cost, that is the difference in response time between maintain trials (the sorting rule stays the same) and switch trials (the sorting rule changes) (Geurts et al., 2009). So far, only two behavioral studies have applied such a switch cost paradigm to investigate cognitive flexibility in ASD and both of them reported intact performance in individuals with ASD.

Taken together, the literature on cognitive flexibility in ASD shows two types of inconsistencies. First, there are inconsistencies within a certain measure: studies using the same task can yield different findings. These inconsistencies might be due to differences in participant characteristics like age, IQ and co-occurring disorders (Geurts et al., 2009; Happé, Booth, Charlton, \& Hughes, 2006; Hill, 2004; Russo et al., 2007). Second, there are inconsistencies between measures. Briefly stated, based on measures of everyday behavior and findings from the WCST, there is clear evidence for impairments in cognitive flexibility in individuals with ASD, whereas studies using other cognitive flexibility tasks generally fail to reveal these impairments. This apparent discrepancy between the obvious inflexibility in natural settings and the inconsistent findings on tasks used in clinical or research settings has been referred to as "the paradox of cognitive flexibility in ASD" (Geurts et al., 2009).

In this article, we propose and test an explanation for the inconsistent findings in the ASD literature on the WCST and the other cognitive flexibility tasks used in clinical or research settings.

One explanation entails that deficits on the WCST are not inherently due to cognitive inflexibility, but result from problems with other aspects of the task (like the high social demands, high working memory and generativity load), that are controlled for in other cognitive flexibility measures. This might indeed explain why individuals with ASD show deficits on the WCST and intact performance on more controlled experimental task switching paradigms. However, it does not explain the intact performance on the other clinical cognitive flexibility tasks that also impose high social demands, and/or high working memory and generativity loads (e.g., the modified WCST and the Playing Cards Test from the Behavioral Assessment of the Dysexecutive Syndrome [BADS]; see Table 1). Furthermore, postulating that individuals with ASD have no cognitive flexibility deficits clearly contradicts with the obvious inflexibility they show in daily life.

Given this clear daily life inflexibility of individuals with ASD, we suggest that they do have problems with cognitive flexibility, but that these deficits only become apparent under specific conditions. When closely comparing the WCST with the other cognitive flexibility tasks, it appears that this task differs from the others in at least one of two factors that might be crucial to elicit the cognitive flexibility deficits in individuals with ASD: (a) the degree of explicitly provided task instructions and (b) the amount of disengagement required to perform the switch.

Cognitive flexibility tasks can be categorized according to the degree of explicitly provided task instructions by applying a five level taxonomy (see Table 1 ). At the lowest level (0), no indications are given about the rules that should be applied, nor that a rule switch will occur. At level 1, participants are instructed before the task that a rule switch will occur. At level 2, participants are instructed that a rule switch will occur and they get an explicit warning during the task indicating when they have to switch. At level 3, participants are instructed before the task what rules should be applied and when to switch to another rule. At the highest level (4), a cue is shown on each trial, explicitly indicating which rule should be applied. Based on this classification, the WCST appears to be the only task with the lowest degree of explicitly provided task instructions. On the contrary, the experimental task-switching paradigms are situated on the highest degree, and all other cognitive flexibility tasks somewhere in between. Our concept of 'degree of explicitly provided task instructions' is comparable with that of 'degree of rule constraints' (Ciesielski \& Harris, 1997) and 'degree of open-endedness' (White, Burgess, \& Hill, 2009). Although these other concepts are broader, we could say that higher degrees of explicitly provided task instructions correspond with higher degrees of rule constraints and lower degrees of open-endedness. Ciesielski and Harris (1997) have already shown that the lower the degree of rule constraints in flexibility tasks, the higher the impairments for individuals 
Overview of cognitive flexibility tasks used in autism research, partially adapted from Geurts et al. (2009).

\begin{tabular}{|c|c|c|c|c|c|}
\hline Task & Description & $\begin{array}{l}\text { Main dependent } \\
\text { measures }\end{array}$ & $\begin{array}{l}\text { ASD versus } \\
\text { TD (\# studies } \\
\text { showing deficient } \\
\text { versus intact } \\
\text { performance) }\end{array}$ & $\begin{array}{l}\text { Degree of } \\
\text { explicitly } \\
\text { provided task } \\
\text { instructions }\end{array}$ & $\begin{array}{l}\text { Disengagement } \\
\text { required? }\end{array}$ \\
\hline $\begin{array}{l}\text { Wisconsin } \\
\text { Card Sorting } \\
\text { Task (WCST) }\end{array}$ & $\begin{array}{l}\text { A sorting task in which participants have to determine how to sort cards on the } \\
\text { basis of unspecified categories (color, form and number). The sorting rules have } \\
\text { to be inferred based on the given feedback. Without notice, the sorting rule } \\
\text { changes after } 10 \text { consecutive correct responses and the participant has to } \\
\text { disengage from the previous sorting rule in order to discover the new rule }\end{array}$ & $\begin{array}{l}\text { - \# or \% perseverative } \\
\text { answers } \\
\text { - \# or \% perseverative } \\
\text { errors } \\
\text { - \# categories found }\end{array}$ & $\begin{array}{l}\text { Deficits: } \\
\text { - Standard WCST }(9: 0)^{\mathrm{a}} \\
\text { - Computerized } \\
\text { WCST }(3: 0)^{\mathrm{b}}\end{array}$ & 0 & Yes \\
\hline Modified WCST & $\begin{array}{l}\text { Similar to the WCST, but now participants are explicitly warned each time when } \\
\text { the rule changes (after six consecutive correct responses). }\end{array}$ & \# perseverative errors & No deficits $(0: 1)^{c}$ & 2 & Yes \\
\hline $\begin{array}{l}\text { Trail Making } \\
\text { Test (TMT) }\end{array}$ & $\begin{array}{l}\text { Timed task consisting of two parts. In part A, a series of numbers has to be } \\
\text { connected in ascending order. In part B, a series of numbers and letters has to be } \\
\text { connected in ascending order while alternating between numbers and letters }\end{array}$ & $\begin{array}{l}\text { - Time B } \\
\text { - Time B - Time A } \\
\text { - Ratio time B/Time A }\end{array}$ & Inconsistent $(2: 2)^{\mathrm{d}}$ & 3 & Yes \\
\hline D-KEFS TMT & $\begin{array}{l}\text { This task consists of five conditions that assess visual scanning, number } \\
\text { sequencing, letter sequencing, number-letter switching and motor speed. The } \\
\text { number-letter switching task requires to switch between connecting numbers } \\
\text { and letters in ascending order }\end{array}$ & $\begin{array}{l}\text { Completion time } \\
\text { switch condition }\end{array}$ & No deficits $(0: 1)^{\mathrm{e}}$ & 3 & Yes \\
\hline $\begin{array}{l}\text { BADS Playing } \\
\text { Cards Test }\end{array}$ & $\begin{array}{l}\text { Playing cards are turned over, one at a time. In the first part, children are } \\
\text { instructed to say 'yes' to red cards and 'no' to black cards. In the second part, the } \\
\text { rule is changed, and children have to indicate whether the card has the same } \\
\text { color as the preceding one }\end{array}$ & $\begin{array}{l}\text { \# errors in the } \\
\text { second part }\end{array}$ & No deficits $(0: 2)^{\mathrm{f}}$ & 3 & Yes \\
\hline $\begin{array}{l}\text { D-KEFS } \\
\text { Color-Word }\end{array}$ & $\begin{array}{l}\text { This task consists of four conditions assessing naming of color patches, reading } \\
\text { of color-words printed in black ink, inhibition and inhibition combined with } \\
\text { switching. In the inhibition condition, participants have to perform the } \\
\text { traditional Stroop task, i.e., inhibiting word reading in order to name the } \\
\text { dissonant ink colors in which the words are printed. In the inhibition/switching } \\
\text { condition, half of the color-words are encased in a box. Participants have to } \\
\text { name the dissonant ink color except for the boxed words, in which case they } \\
\text { must switch and read the word itself }\end{array}$ & $\begin{array}{l}\text { - Completion time } \\
\text { switch condition } \\
\text { - \# errors switch } \\
\text { condition }\end{array}$ & Inconsistent $(1: 1)^{\mathrm{g}}$ & 3 & Yes \\
\hline $\begin{array}{l}\text { CANTAB ID/ } \\
\text { ED Task }\end{array}$ & $\begin{array}{l}\text { On each trial two stimuli are presented and the participant has to learn which } \\
\text { stimulus is correct based on the provided feedback. Nine stages have to be } \\
\text { completed assessing: (1) simple discrimination between two pink shapes; ( } 2 \text { ) } \\
\text { simple reversal, using the same stimuli but with contingencies reversed; ( } 3 \text { ) } \\
\text { compound discrimination separate: the same shape remains correct, but now } \\
\text { the stimuli consist of one of two shapes combined with one of two non- }\end{array}$ & $\begin{array}{l}\text { - \# trials to criterion } \\
\text { at the ED-shift stage } \\
\text { - \# errors to criterion } \\
\text { at the ED-shift stage }\end{array}$ & Inconsistent $(1: 6)^{\mathrm{h}}$ & 1 & $\begin{array}{l}\text { Reduced } \\
\text { disengagement }\end{array}$ \\
\hline
\end{tabular}
the previous stage, but now the lines are superimposed on the shapes (=compound stimuli); (5) compound reversal: using the same stimuli as in 4, but the other shape is correct; (6) intra-dimensional (ID) shift: new compound stimuli are presented and one of the new shapes is correct; (7) intradimensional reversal: using the same stimuli as in 6 , but now the other shape is correct; (8) extra-dimensional (ED) shift: new compound stimuli are presented and now a particular line is correct; (9) extra-dimensional reversal: same stimuli as in 8 , but now the other line becomes correct

or \% perseverative

Ratio time $B /$ Time $A$

Completion time

\# errors in the

Completion tim

\# trials to criterio

the ED-shift stage 
Matching Task

Stimuli have to be sorted on color or shape. Before starting a new block of trials, a cue explicitly indicates the sorting rule. A distinction is made between switch blocks (in which the sorting rule differs from the one in the previous block) and repetition blocks (in which the sorting rule is the same as in the previous block) neuropsychological/experimental measure, and the tasks in the dark grey rows are experimental task-switching paradigms.

a Geurts, Verté, Oosterlaan, Roeyers, and Sergeant (2004), Goldstein, Johnson, and Minshew (2001), Griebling et al. (2010), Lopez et al. (2005), Minshew, Meyer, and Goldstein (2002), Pellicano, Maybery, Durkin, and Maley (2006), Pellicano (2007) and Verté, Geurts, Roeyers, Oosterlaan, and Sergeant $(2005,2006)$

Robinson, Goddard, Dritschel, Wisley, and Howlin (2009), Tsuchiya, Oki, Yahara, and Fujieda (2005) and Winsler, Abar, Feder, Schunn, and Rubio (2007).

${ }^{c}$ Hill and Bird (2006).

d Intact performance: Corbett, Constantine, Hendren, Rocke, and Ozonoff (2009) and Hill \& Bird (2006). Deficient performance:

deficient performance in ASD only looked at the performance on part B, without controlling for group differences on part $\mathrm{A}$

e Lopez et al. (2005).

Hill and Bird (2006) and White et al. (2009).

Intact performance: Lopez et al. (2005). Deficient performance: Corbett et al. (2009).

h Intact performance: Corbett et al. (2009), Goldberg et al. (2005), Happé et al. (2006), Landa and Goldberg (2005), Sinzig, Morsch, Bruning, Schmidt, and Lehmkuhl (2008) and Yerys et al. (2009). Deficient performance: Ozonoff et al. (2004).

${ }^{i}$ Dichter et al. (2010).

j Poljac et al. (2010). 
with ASD compared to typically developing controls. The other factor that may account for the between task inconsistencies in the ASD literature is the amount of disengagement required to perform the rule switch. Typically, switching to another rule requires both disengagement from (or inhibition of) the previously correct stimulus-response association and activation of the required one (Monsell, 2003; Smith, Taylor, Brammer, \& Rubia, 2004). However, in experimental settings the amount of disengagement required to perform a switch can be reduced or even eliminated simply by not showing the previously correct stimulus. This is what happens in the ED-shift of the ID/ED task (see Table 1 for a description of the task). In this stage individuals have to learn a new stimulus-response association (i.e., a new rule), but since the previously correct stimulus is no longer displayed, they do not have to disengage from it. There is evidence that individuals with ASD have problems with disengagement of attention (Casey, Gordon, Mannheim, \& Rumsey, 1993; Hughes \& Russell, 1993; Kawakubo et al., 2007; Landry \& Bryson, 2004). Therefore, reducing the amount of disengagement necessary to perform a switch may facilitate the task for individuals with ASD, hence reducing the differences in performance between individuals with ASD and typically developing controls.

To summarize, as an explanation for the inconsistent findings between the WCST and the other cognitive flexibility tasks we hypothesize that individuals with ASD do show impairments in cognitive flexibility, but that these impairments might only become apparent in situations characterized by the lowest degree of explicitly provided task instructions and a high amount of disengagement required to perform the switch.

So far, the WCST is the only task fulfilling both requirements. However, this task requires various additional cognitive processes besides switching, making it uncertain whether difficulties are due to cognitive flexibility impairments. The aim of this study is to investigate whether individuals with ASD show cognitive flexibility impairments on a more controlled, but equally open-ended task-switching variant of the WCST. We therefore developed a task-switching paradigm with the following characteristics: (a) the task has the lowest degree of explicitly provided task instructions ( WCST); (b) the task requires a high amount of disengagement to perform the switch ( $\sim$ WCST); but (c) the influence of confounding variables on task performance is minimized on the one hand by reducing social demands, working memory and generativity load, and on the other hand by providing a within-subject calculation of the switch cost. This version of the WCST is an adaptation of a task used to investigate brain correlates of cognitive flexibility in typically developing participants (Watson, Azizian, \& Squires, 2006). In the present study, the task was administered to a group of high-functioning children with ASD and age- and IQ-matched typically developing controls. Consistent with our hypothesis, we predict that children with ASD will show more perseveration errors and a higher switch cost on this task compared to typically developing controls.

\section{Methods}

\subsection{Participants}

The autism spectrum disorder (ASD) group comprised 40 children (36 boys and 4 girls). They all received a formal diagnosis of ASD made by a multidisciplinary team according to DSM-IV-TR criteria (APA, 2000). Twenty-one of them were recruited through the Flemish Autism Association. The 19 other children participated in a larger family study of the Leuven Autism Research (LAuRes) consortium. Their diagnosis was additionally confirmed with the Developmental, Dimensional and Diagnostic Interview (3di; Skuse et al., 2004). Both ASD groups did not differ significantly from one another on IQ, nor on test performance (perseveration errors and switch cost; data not shown). To increase power they were collapsed into an omnibus ASD group. None of the ASD children had a known neurological or genetic disorder.

The control group comprised 40 typically developing (TD) children (36 boys and 4 girls), recruited through schools, personal contacts and advertisements. None of these children presented any neurological or psychiatric disorders or had a first degree family member with a developmental, learning or neurological disorder. None of these children was on medication. This information was gathered during a telephone conversation with one of the parents.

We included children and adolescents from 8 to 18 years old, with a verbal, performance, and total IQ score above 70 . Intelligence was assessed with a shortened version of the Wechsler Intelligence Scale for Children, Third Edition (WISC-III; Wechsler, 1992), or with a shortened version of the Wechsler Adult Intelligence Scale, Third Edition (WAIS-III; Wechsler, 1997) for participants above 17 years old. This shortened version consisted of four subtests: Vocabulary, Similarities, Picture Completion and Block Design (Sattler, 2001). The participants were group-wise matched on the basis of gender, chronological age, verbal IQ, performance IQ and full-scale IQ (see Table 2).

Table 2

Characteristics of the participating groups.

\begin{tabular}{|c|c|c|c|c|c|c|}
\hline \multirow[t]{2}{*}{ Characteristics } & \multicolumn{2}{|c|}{ ASD group $(n=40)$} & \multicolumn{2}{|c|}{ TD group $(n=40)$} & \multirow[t]{2}{*}{$t(78)$} & \multirow[t]{2}{*}{$p$} \\
\hline & Mean & SD & Mean & SD & & \\
\hline Age & 11.33 & 2.18 & 11.13 & 2.22 & 0.41 & 0.69 \\
\hline VIQ & 106.68 & 14.82 & 109.65 & 12.14 & 0.98 & 0.33 \\
\hline PIQ & 104.25 & 14.77 & 103.88 & 14.09 & 0.12 & 0.91 \\
\hline FSIQ & 105.45 & 12.34 & 106.76 & 9.04 & 0.54 & 0.59 \\
\hline
\end{tabular}




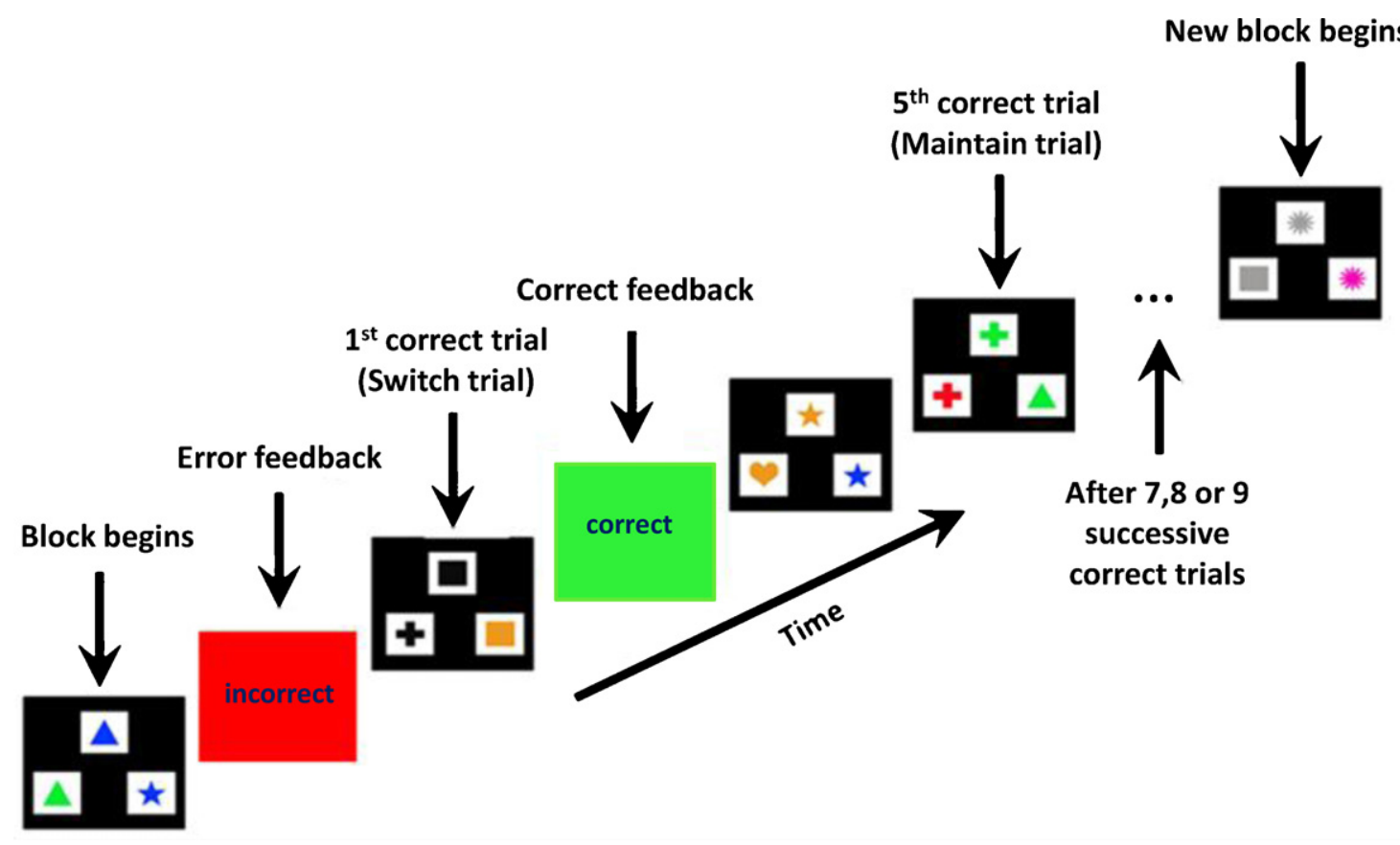

Fig. 1. Illustration of the protocol of the Wisconsin Card Sorting Task With Controlled Task Switching (WCST-WCTS).

\subsection{Materials: Wisconsin Card Sorting Task With Controlled Task Switching (WCST-WCTS)}

For this task, children sat approximately $57 \mathrm{~cm}$ from a 17 -in. LCD computer screen on which the stimuli were displayed. On each trial, three cards arranged in a pyramid form were simultaneously presented on the screen (see Fig. 1). Each card contains a figure with a specific shape (circle, triangle, heart, cross, star, square, lightning or flower) and color (blue, green, yellow, red, grey, pink, black or orange). The card at the top is the reference card, and the cards at the bottom are choice cards. One of the two choice cards has the same shape as the reference card, but has a different color. The other choice card has the same color as the reference card, but has a different shape. Children had to match the reference card with the correct choice card, based on either color or shape. Only two sorting rules from the original WCST were implemented to minimize the working memory demands (Watson et al., 2006). On two successive trials the cards had different colors and different shapes. The sorting stimulus (consisting of the three cards) remained on the screen until participants made a valid response. Responses were made by pressing the left or right button of a 2-button-response box. Both accuracy and reaction time were recorded. For half of the trials the correct choice card was the left one and required a left button press, and for the other half the correct choice card was the right one and required a right button press. After responding, visual and auditory feedback was given during $600 \mathrm{~ms}$ (the word "correct" displayed on a green background accompanied by a high tone versus the word "incorrect" on a red background accompanied by a low tone). Stimulus presentation and response registration were controlled by Affect 4.0 (Spruyt, Clarysse, Vansteenwegen, Baeyens, \& Hermans, 2010).

At the beginning of the task participants received the following instructions: "In this task three cards are shown. One card appears at the top and two cards appear at the bottom, one left and one right. You have to indicate whether the upper card matches the left or right card at the bottom, by pressing the left or right response button as soon as possible. The computer will then indicate whether your choice was correct or incorrect. You have to try to correctly sort as many cards as possible, as fast as possible. Is that clear?". The examiner was not allowed to give any indication that the sorting principles involve color or shape, or that there would be a switch from one category to the other. After instructions, a practice block was completed to ensure that the participant understood the instructions. In this practice block cards had to be sorted according to shape and after eight consecutive correct responses this block was completed. After the practice block, the participants were presented with five runs, each comprising five blocks. In each block a particular sorting rule had to be applied. Randomly after seven, eight or nine consecutive correct answers the sorting rule changed and a new block began. Accordingly, the sorting rule changed four times in each run and 20 times throughout the whole task. After each run a short break was provided to avoid fatigue. Failure to achieve the criterion of 7-9 consecutive correct responses within 50 trials resulted in discontinuation of the task.

The main parameters of interest were the mean number of perseveration errors and the switch cost. A perseveration error is defined as the repetition of an incorrect answer. To account for task discontinuation, we controlled for the number of completed blocks by calculating the mean number of perseveration errors per block. The switch cost is calculated as the difference between the mean reaction time on switch trials and the mean reaction time on maintain trials. A switch trial is 
defined as the first correct trial of a block, following an incorrect trial and followed by four consecutive correct answers. A maintain trial is defined as the fourth correct trial after a switch trial. For each block, except the first block of each run, one switch and one maintain trial were calculated (see Fig. 1).

Additionally, the number of practice trials required to complete the practice block was registered as it provides a general indication of the ability to learn a rule from feedback. After the task, participants were also asked which sorting rules they had to apply, to check whether the correct rules (color and shape) were used.

Finally, a number of other parameters were also derived because they are also measured by other commonly used flexibility tasks, like the ID/ED task and the WCST: (a) the number of blocks completed; (b) the mean number of trials per block: the mean number of trials necessary to attain the criterion of 7-9 consecutive correct responses; (c) the mean number of failures to maintain set: the number of times the participant makes an error after five consecutive correct answers, divided by the number of blocks completed; and (d) the mean number of errors: the sum of the mean number of perseveration errors and the mean number of failures to maintain set.

\subsection{Procedure}

Informed consent was obtained from the parents and also from the participants older than 16 years. All participants were tested individually in a quiet room either at the University Hospital 'Gasthuisberg' in Leuven, or at school. Testing took place within the context of a larger study consisting of two 2-h sessions. The order of the tasks was counterbalanced to avoid ordereffects. In addition, computerized tasks were altered with other task formats, to provide enough variation. Furthermore, breaks were provided to avoid fatigue. After testing, participants could choose a reward for their participation (e.g., a film ticket, a comic book, marbles, a ball, etc.).

This study was approved by the Medical Ethical Committee of the University Hospitals Leuven and the Ethical Committee of the Faculty of Psychology and Educational Sciences of the Katholieke Universiteit Leuven, Belgium.

\subsection{Data analysis}

For the reaction time data, all values smaller than $100 \mathrm{~ms}$ or larger than $5000 \mathrm{~ms}$ were removed. Afterwards, data were inspected for outliers on the main dependent variables (mean perseveration errors and switch cost). A participant was considered an outlier if his/her standard score was $\geq 2.5$ above or below the mean on at least one of these variables. This yielded one outlier per group for the mean perseveration errors and three outliers per group for the switch cost. All the analyses were performed including and excluding these outliers. Both ways of analyzing yielded similar results, although group differences were more pronounced when the outliers were excluded. Here we only report the analysis on the full sample as it is more conservative and representative.

The assumptions for parametric testing were checked and appeared to be violated for all dependent measures. To obtain more normally distributed data, the reaction time measures were log-transformed prior to analysis. For the other measures, non-parametric Mann-Whitney $U$ tests were applied. A significance level of $p<0.05$ (two-sided) was adopted for all analyses and Cohen's $d$ effect sizes were calculated for between-subject comparisons. An effect size ranging from 0.2 to 0.3 is considered small, values around 0.5 are medium and values of 0.8 or above are considered large effects (Cohen, 1988).

\section{Results}

The main cognitive flexibility measures were the mean number of perseveration errors per block and the switch cost.

The mean number of perseveration errors was higher for the ASD group than for the TD group (see Fig. 2). In spite of the considerable effect size $(d=0.53)$, this difference was only marginally significant (Mann-Whitney $U=1792 ; p=0.09$ ). In addition to the conservative way of testing this effect, this lack of significance is probably due to the large variance in the ASD group, which was significantly larger than the variance in the TD group (Levene's test for homogeneity: $F(1,78)=4.91$; $p=0.03)$.

To evaluate the switch cost, a $2 \times 2$ (trial type $\times$ group) repeated measure analysis of variance (ANOVA) was performed, with reaction time (RT) as the dependent measure, trial type (switch versus maintain trials) as the within-subject factor and group as the between-subject factor (see Fig. 3). This analysis revealed a significant main effect of trial type $(F(1,78)=362.33$, $p<0.001$, with slower reaction times on switch than on maintain trials), no main effect of group $(F(1,78)=2.86, p=0.09)$, and a significant trial type $\times$ group interaction $(F(1,78)=4.90, p=0.03)$. Follow-up contrasts revealed no significant group difference on the maintain trials $(t(78)=1.27, p=0.21, d=0.12)$, but a highly significant group difference on the switch trials $(t(78)=4.40, p<0.001, d=0.52)$. In addition to this repeated measure analysis, the switch cost was calculated for each individual (i.e., the mean reaction time on switch trials minus the mean reaction time on maintain trials) and both groups were compared on this measure (see Fig. 4). A t-test revealed that the ASD group presented a significantly higher switch cost than the TD group $(t(78)=2.64, p=0.01, d=0.60)$.

Both groups were also compared on the additional measures but no significant group differences were found (see Table 3 ). Furthermore, after the task all participants confirmed that they understood the task requirements, i.e., that they had to switch between sorting according to color and sorting according to shape. 


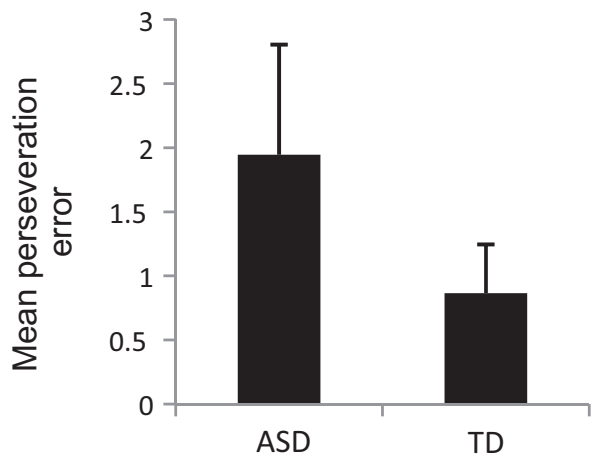

Fig. 2. Mean number of perseveration errors per block for children with an autism spectrum disorder (ASD) and typically developing controls (TD). Error bars depict $1 \mathrm{SE}$ of the mean.

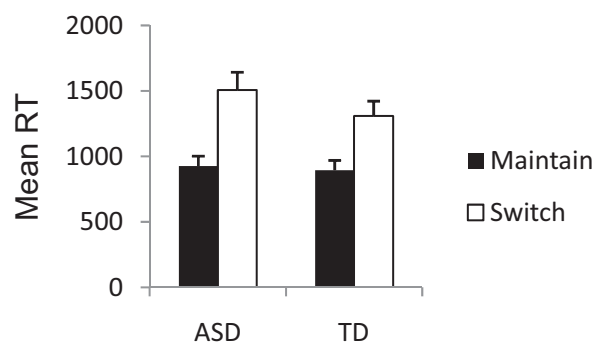

Fig. 3. Mean reaction time as a function of group (ASD versus TD) and trial type (switch versus maintain trials). Error bars depict 1 SE of the mean.

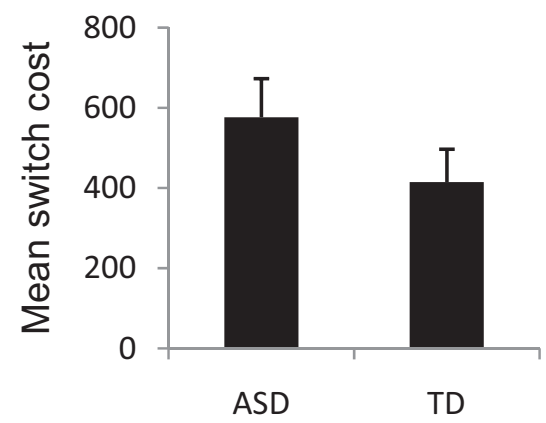

Fig. 4. Mean switch cost of the ASD and the TD group. Error bars depict $1 \mathrm{SE}$ of the mean.

Table 3

Group comparison for the additional measures.

\begin{tabular}{llllrl}
\hline Measure & ASD group $(n=40)$ & TD group $(n=40)$ & Mann-Whitney $U$ & $p$ (two-sided) \\
& Mean (SD) & Cohen's $d$ \\
\hline Number of practice trials & $12.58(8.37)$ & $10.35(3.83)$ & 1773.5 & 0.12 & 0.34 \\
Number of blocks completed & $19.33(8.98)$ & $21.83(6.99)$ & 1521 & 0.22 & 0.24 \\
Trials per block & $17.48(6.79)$ & $15.15(4.63)$ & 1743 & 0.58 & 0.40 \\
Mean maintain failures per block & $0.31(0.23)$ & $0.36(0.32)$ & 1561.5 & 1756.5 & 0.18 \\
Total errors & $2.26(2.73)$ & $1.23(1.33)$ & & 0.47 \\
\hline
\end{tabular}

\section{Discussion}

The present study investigated the performance of children with ASD and typically developing controls on an experimental task-switching variant of the WCST, which controls for confounding variables, requires a high amount of disengagement to perform the switch, and has the lowest degree of explicitly provided task instructions. (According to our taxonomy outlined in Section 1 this means that there are no indications that a switch will occur, nor about the rules that should be applied.) Consistent with the task-switching literature, all participants responded slower on switch than on maintain trials, showing that this task induced a switch cost (Monsell, 2003). When comparing the performance of the ASD and the TD groups, the data revealed that children with ASD tended to make more perseveration errors and had a higher 
switch cost compared to typically developing controls. This is consistent with our predictions and indicates cognitive flexibility impairments in children with ASD. In addition, there was no group difference in the number of practice trials needed to complete the practice block, implying that both groups understood the task instructions and were equally able to learn a rule from feedback. Furthermore, when asked which sorting rules they had to apply, all participants correctly indicated that they had to switch between sorting according to color and sorting according to shape, showing that they used the correct sorting rules. Individuals with ASD neither had problems to maintain a certain rule. This is evidenced by comparable mean numbers of failures to maintain set between both groups. In addition, the reaction time data revealed that both groups responded equally fast on maintain trials. However, children with ASD responded significantly slower on the switch trials. All these findings indicate that individuals with ASD had specific difficulties with switching, but not with other aspects of the task.

Although the mean number of perseveration errors was considerably higher for the ASD group compared to the TD group (and the effect size was fairly large), this difference was not significant. A closer inspection of the results indicates that this might be due to the large variance in the ASD group, which was significantly larger than that of the TD group. This group difference in variance might be due to floor effects in the control group or to the larger heterogeneity in the ASD group.

Some cognitive flexibility tasks, like the ID/ED task and the WCST, use other indicators of cognitive flexibility: the number of blocks completed, the number of trials per block and the number of total errors. When comparing the performance of both groups on these measures, no group differences were found. Since group differences were found on switch cost measures, this might indicate that the other measures are not sensitive enough to detect cognitive flexibility deficits. For example, the number of total errors is the sum of the perseveration errors and the failures to maintain set. Our data indicate that the ASD group made more perseveration errors, but slightly less failures to maintain set compared to the TD group. So in this case, the group difference for the number of total errors is smaller, and thus less sensitive to detect differential performance, than the number of perseveration errors.

In this study, we administered a task-switching paradigm with the lowest degree of explicitly provided task instructions and found flexibility impairments in children with ASD. The only other cognitive flexibility task with this degree of explicitly provided task instructions is the WCST, which also reveals flexibility deficits in individuals with ASD (see Table 1). However, all other task-switching paradigms currently used in ASD research have high degrees of explicitly provided task instructions and do not reveal cognitive flexibility impairments in individuals with ASD. This corroborates our hypothesis that the degree of explicitly provided task instructions might be crucial to elucidate cognitive flexibility impairments in ASD. In line with this, it has been demonstrated that tasks with low degrees of 'rule constraints' (Ciesielski \& Harris, 1997) or more openended tasks (White et al., 2009) provoke the most severe impairments in individuals with ASD.

The degree of explicitly provided task instructions can also be related to different degrees of internally versus externally controlled shifting. Based on a factor analysis, it has been shown that two separate groups of cognitive flexibility tasks can be discerned: tasks requiring self-directed or internal control over shifting and tasks reflecting external control over shifting (Teunisse, Cools, van Spaendonck, Aerts, \& Berger, 2001). Tasks with low degrees of explicitly provided rule constraints rely more on internally controlled processing because of the implicit shifting rules and categories, whereas the explicitly presented shifting rules elicit more externally controlled shifting behavior (Teunisse et al., 2001). In line with this, Gioia et al. (2002) mention that in tests tapping executive functions in an explicit way (thus with high degrees of explicitly provided task instructions) the examiner provides the necessary structure and organization, thereby serving as the participant's external executive control and relieving the demands on executive functions (including cognitive flexibility). They further state that, as a result, an individual with significant executive dysfunctions can often perform adequately on well-structured tests (Gioia et al., 2002). This is evinced by a lesion study demonstrating that performance on the WCST of patients with focal frontal lesions improved when they were informed about the sorting rules and received a warning during the task indicating that the rule would change on the following trial (without mentioning the actual sorting criterion) (Stuss et al., 2000). Accordingly Stuss et al. (2000) demonstrated that explicitly providing structured verbal instructions makes a test less sensitive to frontal lesions. Based on these findings, it is plausible that individuals with ASD do present cognitive flexibility impairments, but that they are able to compensate for their deficits on tasks with high degrees of explicitly provided task instructions. Support for this interpretation is provided by an fMRI study comparing performance of individuals with ASD and TD controls on a switch task with a cue presented before each trial, explicitly indicating which rule should be applied (Schmitz et al., 2006). Both groups had similar behavioral results, but individuals with ASD showed increased parietal lob activation. According to Schmitz et al. (2006, p. 14), this "might reflect a compensatory mechanism for dysfunctional frontal brain regions". The idea that offering structure might reduce cognitive flexibility deficits has important implications as it underscores the usefulness of intervention strategies for ASD that emphasize the explicit provision of structure and explicit step-by-step instructions (Klin \& Volkmar, 1995).

Cognitive flexibility deficits of individuals with ASD on more open-ended tasks might be due to difficulties with internal, more abstract regulation of performance or problems with understanding the implicit task demands (Larson et al., 2010; White et al., 2009). These deficits can also be linked with performance monitoring difficulties. Performance monitoring (also referred to as response monitoring) involves the process of evaluating the consequences of behavior and making adjustments to optimize outcomes (Thakkar et al., 2008). This appears to be problematic for individuals with ASD (South, Larson, Krauskopf, \& Clawson, 2010; Thakkar et al., 2008). Difficulties with performance monitoring in ASD have been proposed to result from structural and functional abnormalities in the anterior cingulate cortex (ACC), which were also related to higher ratings of repetitive behavior (Thakkar et al., 2008). Given that individuals with ASD perform adequately on 
explicitly cued switching tasks but fail on the more implicit switching tasks, where they have to interpret their own errors as a cue for initiating the switch, we hypothesize that a core aspect of the cognitive flexibility impairments in ASD might rely on deficient monitoring, interpreting and adjusting their own behavior. Further research is needed to investigate the links between cognitive flexibility deficits, problems with performance monitoring, and repetitive behavior in individuals with ASD.

Concerning the issue of disengagement required to perform a switch, there is evidence that individuals with ASD have problems with attentional disengagement (Casey et al., 1993; Hughes \& Russell, 1993; Kawakubo et al., 2007; Landry \& Bryson, 2004). However our study does not allow for disentangling the relative importance of disengagement versus explicit task instructions for revealing cognitive flexibility deficits in ASD. It is also unclear whether tasks need to have both the lowest degree of explicitly provided rule constraints as well as high amounts of disengagement in order to elicit a cognitive flexibility deficit in ASD. This study only demonstrates that children with ASD with IQ scores above 70, are impaired on a task switching variant of the WCST with the lowest degree of explicitly provided task instructions and high amounts of disengagement required to perform the switch. Although there is additional evidence supporting our hypothesis that both the degree of explicitly provided task instructions and the amount of disengagement might be crucial to elucidate cognitive flexibility impairments in ASD (see Section 1), further research is needed to establish this more firmly. This can be done, for example, by directly comparing performance on tasks with low versus high degrees of explicitly provided task instructions and low versus high degrees of disengagement required to perform the switch in a $2 \times 2$ factorial within-subjects design. An additional question that could be addressed in further research is whether our findings can be generalized to individuals with ASD with a different age and/or IQ range. An important remark in this regard is that individuals with ASD are characterized by a large heterogeneity both in the ASD phenotype (Wing, 1997) and in neurocognitive characteristics. Therefore, it might be possible that several more homogeneous subgroups exist, each with a specific cognitive flexibility profile.

A last remark concerns the ecological validity of the cognitive flexibility measures used in experimental studies. In daily life, flexibly switching to different thoughts or actions is mostly triggered implicitly by events indicating that alternative thoughts or actions may be more appropriate given the situation. In addition, in order to effectively perform a switch the individual mostly has to disengage attention from the current thoughts or actions. Although all cognitive flexibility tasks greatly reduce the characteristics of daily life situations, we do suggest that more open-ended and implicit tasks (compared to highly constrained and explicit tasks), as well as tasks requiring high amounts of disengagement, more closely resemble everyday situations and are therefore more ecologically valid. This is supported by the finding that measures of everyday behavior (e.g., the BRIEF, Gioia et al., 2002; Mackinlay et al., 2006) as well as the most open-ended and implicit flexibility tasks that require high amounts of disengagement (the WCST and our task) reveal cognitive flexibility impairments in ASD, while all other cognitive flexibility tasks fail to consistently show these deficits (see also Table 1). Additional research might further investigate the ecological validity of these tasks by calculating the correlation between task performance and the cognitive flexibility score of the BRIEF.

In summary, the present study investigated the performance of children with ASD and typically developing controls on an experimental task-switching variant of the WCST, which controls for confounding variables, requires a high amount of disengagement to perform the switch, and has the lowest degree of explicitly provided task instructions. As predicted, individuals with ASD made more perseveration errors and had a higher switch cost than typically developing controls, but they performed equally well on the control measures. These findings, along with other observations, indicate that individuals with ASD do have cognitive flexibility impairments, but that these impairments might only be revealed under conditions with a low degree of explicitly provided task instructions and a high amount of disengagement required to perform the switch. However, further research is needed to fully establish this claim.

\section{Acknowledgements}

This study was funded by a doctoral and postdoctoral fellowship from the Fund for Scientific Research (FWO Flanders) to Lien Van Eylen and Bart Boets, respectively, a grant from the Research Council of the K.U. Leuven (IDO/08/013) to Jean Steyaert, Johan Wagemans, and Ilse Noens, and from the Methusalem program (METH/08/02) to Johan Wagemans. The authors thank all children who participated, Jeroen Clarysse for his help with programming the task in Affect 4.0, and the following master students from the faculty of Psychology and Educational Sciences for their assistance in data collection: Jolien Hoskens, Carola Damiaans, Ioanna Kitsinis, Mieke Boes, Eline De Proft, and Chiara Boodts.

\section{References}

American Psychiatric Association. (2000). Diagnostic and statistical manual of mental disorders (4th ed.). Washington, DC: Author.

Boyd, B. A., McBee, M., Holtzclaw, T., Baranek, G. T., \& Bodfish, J. W. (2009). Relationships among repetitive behaviors, sensory features, and executive functions in high functioning autism. Research in Autism Spectrum Disorders, 3, 959-966.

Casey, B. J., Gordon, C. T., Mannheim, G. B., \& Rumsey, J. M. (1993). Dysfunctional attention in autistic savants. Journal of Clinical and Experimental Neuropsychology, 15, 933.

Ciesielski, K., \& Harris, R. J. (1997). Factors related to performance failure on executive tasks in autism. Child Neuropsychology, 3, 1-12.

Cohen, J. (1988). Statistical power analysis for the behavioral sciences (2nd ed.). Hillsdale, NJ: Lawrence Erlbaum Associates.

Corbett, B. A., Constantine, L. J., Hendren, R., Rocke, D., \& Ozonoff, S. (2009). Examining executive functioning in children with autism spectrum disorder, attention deficit hyperactivity disorder and typical development. Psychiatry Research, 166, 210-222. 
Denckla, M. B. (1996). A theory and model of executive function: A neuropsychological perspective. In G. R. Lyon \& N. A. Krasnegor (Eds.), Attention, memory, and executive function (pp. 263-277). Baltimore, MD: Paul H. Brookes.

Dichter, G. S., Radonovich, K. J., Turner-Brown, L. M., Lam, K. S. L., Holtzclaw, T. N., \& Bodfish, J. W. (2010). Performance of children with autism spectrum disorders on the Dimension-Change Card Sort task. Journal of Autism and Developmental Disorders, 40, 448-456.

Geurts, H. M., Verté, S., Oosterlaan, J., Roeyers, H., \& Sergeant, J. A. (2004). How specific are executive functioning deficits in attention deficit hyperactivity disorder and autism? Journal of Child Psychology E Psychiatry, 45, 836-854.

Geurts, H. M., Corbett, B., \& Solomon, M. (2009). The paradox of cognitive flexibility in autism. Trends in Cognitive Sciences, $13,74-82$.

Gioia, G. A., Isquith, P. K., Kenworthy, L., \& Barton, R. M. (2002). Profiles of everyday executive function in acquired and development disorders. Child Neuropsychology, 8, 121-137.

Goldberg, M., Mostofsky, S. H., Cutting, L. E., Mahone, E. M., Astor, B. C., Denckla, M. B., et al. (2005). Subtle executive impairment in children with autism and children with ADHD. Journal of Autism and Developmental Disorders, 35, 279-293.

Goldstein, G., Johnson, C. R., \& Minshew, N. J. (2001). Attentional processes in autism. Journal of Autism and Developmental Disorders, 31 , 433.

Griebling, J., Minshew, N., Bodner, K., Libove, R., Bansal, R., Konasale, P., et al. (2010). Dorsolateral prefrontal cortex magnetic resonance imaging measurements and cognitive performance in autism. Journal of Child Neurology, 25, 856-863.

Happé, F., \& Ronald, A. (2008). The fractionable autism triad: A review of evidence from behavioural, genetic, cognitive and neural research. Neuropsychology Review, 18, 287-304.

Happé, F., Booth, R., Charlton, R., \& Hughes, C. (2006). Executive function deficits in autism spectrum disorders and attention-deficit/hyperactivity disorder: Examining profiles across domains and ages. Brain and Cognition, 61, 25-39.

Hill, E. L. (2004). Evaluating the theory of executive dysfunction in autism. Developmental Review, 24, 189-233.

Hill, E. L., \& Bird, C. M. (2006). Executive processes in Asperger syndrome: Patterns of performance in a multiple case series. Neuropsychologia, 44, 2822-2835.

Hughes, C., \& Russell, J. (1993). Autistic children's difficulty with mental disengagement from an object: Its implications for theories of autism. Developmental Psychology, 29, 498-510.

Kawakubo, Y., Kasai, K., Okazaki, S., Hosokawa-Kakurai, M., Watanabe, K., Kuwabara, H., et al. (2007). Electrophysiological abnormalities of spatial attention in adults with autism during the gap overlap task. Clinical Neurophysiology, 118, 1464-1471.

Klin, A., \& Volkmar, F. (1995). Asperger's syndrome: Guidelines for treatment and intervention. New Haven, Connecticut: Learning Disabilities Association of America.

Landa, R. J., \& Goldberg, M. C. (2005). Language, social, and executive functions in high functioning autism: A continuum of performance. Journal of Autism and Developmental Disorders, 35, 557-573.

Landry, R, \& Bryson, S. E. (2004). Impaired disengagement of attention in young children with autism. Journal of Child Psychology and Psychiatry, 45, 1115-1122.

Larson, M. J., South, M., Krauskopf, E., Clawson, A., \& Crowley, M. J. (2010). Feedback and reward processing in high-functioning autism. Psychiatry Research, in press, doi:10.1016/j.psychres.2010.11.006.

Lopez, B. R., Lincoln, A. J., Ozonoff, S., \& Lai, Z. (2005). Examining the relationship between executive functions and restricted, repetitive symptoms of autistic disorder. Journal of Autism and Developmental Disorders, 35, 445-460.

Mackinlay, R, Charman, T., \& Karmiloff-Smith, A. (2006). High functioning children with autism spectrum disorder: A novel test of multitasking. Brain and Cognition, 61, 14-24.

Minshew, N. J., Meyer, J., \& Goldstein, G. (2002). Abstract reasoning in autism: A disassociation between concept formation and concept identification. Neuropsychology, 16, 327-334.

Monsell, S. (2003). Task switching. Trends in Cognitive Sciences, 7, 134-140.

Ozonoff, S., Cook, I., Coon, H., Dawson, G., Joseph, R. M., Klin, A., et al. (2004). Performance on Cambridge neuropsychological test automated battery subtests sensitive to frontal lobe function in people with autistic disorder: Evidence from the collaborative programs of excellence in autism network. Journal of Autism and Developmental Disorders, 34, 139-150.

Pellicano, E. (2007). Links between theory of mind and executive function in young children with autism: Clues to developmental primacy. Developmental Psychology, 43, 974-990.

Pellicano, E., Maybery, M., Durkin, K., \& Maley, A. (2006). Multiple cognitive capabilities/deficits in children with an autism spectrum disorder: Weak central coherence and its relationship to theory of mind and executive control. Development and Psychopathology, 18, 77-98.

Pennington, B. F., \& Ozonoff, S. (1996). Executive functions and developmental psychopathology. Journal of Child Psychology and Psychiatry and Allied Disciplines, 37, 51-87.

Poljac, E., Simon, S., Ringlever, L., Kalcik, D., Groen, W. B., Buitelaar, J. K., et al. (2010). Impaired task switching performance in children with dyslexia but not in children with autism. The Quarterly Journal of Experimental Psychology, 63, 401.

Robinson, S., Goddard, L., Dritschel, B., Wisley, M., \& Howlin, P. (2009). Executive functions in children with autism spectrum disorders. Brain and Cognition, 71, 362-368.

Russo, N., Flanagan, T., Iarocci, G., Berringer, D., Zelazo, P. D., \& Burack, J. A. (2007). Deconstructing executive deficits among persons with autism: Implications for cognitive neuroscience. Brain and Cognition, 65, 77-86.

Sattler, J. M. (2001). Assessment of children: Cognitive applications (4th ed.). San Diego, CA: Jerome M Sattler Publisher Inc

Schmitz, N., Rubia, K., Daly, E., Smith, A., Williams, S., \& Murphy, D. G. (2006). Neural correlates of executive function in autistic spectrum disorders. Biological Psychiatry, 59, 7-16.

Sinzig, J., Morsch, D., Bruning, N., Schmidt, M. H., \& Lehmkuhl, G. (2008). Inhibition, flexibility, working memory and planning in autism spectrum disorders with and without comorbid ADHD-symptoms. Child and Adolescent Psychiatry and Mental Health 2 Retrieved from http://www.capmh.com/content/2/1/4.

Skuse, D., Warrington, R., Bishop, D., Chowdhury, U., Lau, J., Mandy, W., et al. (2004). The developmental, dimensional and diagnostic interview (3di): A novel computerized assessment for autism spectrum disorders. Journal of the American Academy of Child and Adolescent Psychiatry, 43, 548-558.

Smith, A. B, Taylor, E., Brammer, M., \& Rubia, K. (2004). Neural correlates of switching set as measured in fast, event-related functional magnetic resonance imaging. Human Brain Mapping, 21, 247-256.

South, M., Ozonoff, S., \& McMahon, W. M. (2007). The relationship between executive functioning, central coherence, and repetitive behaviors in the highfunctioning autism spectrum. Autism, 11, 437-451.

South, M., Larson, M. J., Krauskopf, E., \& Clawson, A. (2010). Error processing in high-functioning autism spectrum disorders. Biological Psychology, 85, 242-251.

Spruyt, A., Clarysse, J., Vansteenwegen, D., Baeyens, F., \& Hermans, D. (2010). Affect 4.0. Experimental Psychology, 57, 36-45.

Stuss, D. T., Levine, B., Alexander, M. P., Hong, J., Palumbo, C., Hamer, L., et al. (2000). Wisconsin Card Sorting Test performance in patients with focal frontal and posterior brain damage: Effects of lesion location and test structure on separable cognitive processes. Neuropsychologia, 38, 388-402.

Teunisse, J., Cools, A. R., van Spaendonck, K. P., Aerts, F. H., \& Berger, H. J. (2001). Cognitive styles in high-functioning adolescents with autistic disorder. Journal of Autism and Developmental Disorders, 31, 55-66.

Thakkar, K. N., Polli, F. E., Joseph, R. M., Tuch, D. S., Hadjikhani, N., Barton, J. J., et al. (2008). Response monitoring, repetitive behaviour and anterior cingulate abnormalities in autism spectrum disorders (ASD). Brain, 131, 2464-2478.

Tsuchiya, E., Oki, J., Yahara, N., \& Fujieda, K. (2005). Computerized version of the Wisconsin card sorting test in children with high-functioning autistic disorder or attention-deficit/hyperactivity disorder. Brain and Development, 27, 233-236.

Turner, M. (1997). Towards an executive dysfunction account of repetitive behavior in autism. In J. Russell (Ed.), Autism as an executive disorder. New York, US: Oxford University Press.

Verté, S., Geurts, H. M., Roeyers, H., Oosterlaan, J., \& Sergeant, J. A. (2005). Executive functioning in children with autism and Tourette syndrome. Development and Psychopathology, 17, 415-445.

Verté, S., Geurts, H. M., Roeyers, H., Oosterlaan, J., \& Sergeant, J. A. (2006). Executive functioning in children with an autism spectrum disorder: Can we differentiate within the spectrum? Journal of Autism and Developmental Disorders, 36, 351-372. 
Watson, T. D., Azizian, A., \& Squires, N. K. (2006). Event-related potential correlates of extradimensional and intradimensional set-shifts in a modified Wisconsin Card Sorting Test. Brain Research, 1092, 138-151.

Wechsler, D. (1992). Wechsler intelligence scale for children (3rd ed.). London: The Psychological Corporation.

Wechsler, D. (1997). Wechsler adult intelligence scale (3rd ed.). San Antonia, TX: The Psychological Corporation.

White, S. J., Burgess, P. W., \& Hill, E. L. (2009). Impairments on open-ended executive function tests in autism. Autism Research, 2, 138-147.

Wing, L. (1997). The autism spectrum. The Lancet, 350, 1761-1766.

Winsler, A., Abar, B., Feder, M., Schunn, C., \& Rubio, D. (2007). Private speech and executive functioning among high-functioning children with autistic spectrum disorders. Journal of Autism and Developmental Disorders, 37, 1617-1635.

Yerys, B. E., Wallace, G. L., Harrison, B., Celano, M. J., Giedd, J. N., \& Kenworthy, L. E. (2009). Set-shifting in children with autism spectrum disorders. Autism, 13, 523538. 